\title{
Comparison of Two Predictive Scores for the Development of Incisional Hernia
}

\author{
Edgard Efren Lozada Hernández ${ }^{1, *}$, Miguel Agustín González Gonzalez², \\ José Francisco Molina Rodríguez ${ }^{3}$, Enrique Obregón Moreno ${ }^{4}$, Aldo Edyair Jiménez Herevia ${ }^{4}$, \\ Martin Cano Rosas ${ }^{4}$
}

${ }^{1}$ Surgery Department and Clinical Research, Regional Hospital of High Specialty Bajio, Leon Guanajuato, Mexico

${ }^{2}$ Surgery Department, General Hospital of Calvillo, Aguascalientes, Mexico

${ }^{3}$ Surgery Department, Juarez Hospital of Mexico, Mexico City, Mexico

${ }^{4}$ Surgery Department, Regional Hospital of High Specialty Bajio, Leon Guanajuato, Mexico

Email address:

edgardlozada@hotmail.com (E. E. L. Hernández), mdmiguel_glezglez@outolook.com (M. A. G. Gonzalez), pacomolina89@hotmail.com (J.F.M. Rodríguez), e_obregon_m@hotmail.com (E. O. Moreno), aldojmzherevia@hotmail.com (A. E. J. Herevia),drmartincano@gmail.com (M. C. Rosas)

${ }^{*}$ Corresponding author

\section{To cite this article:}

Edgard Efren Lozada Hernández, Miguel Agustín González Gonzalez, José Francisco Molina Rodríguez, Enrique Obregón Moreno, Aldo Edyair Jiménez Herevia, Martin Cano Rosas. Comparison of Two Predictive Scores for the Development of Incisional Hernia. Journal of Surgery. Vol. 7, No. 6, 2019, pp. 188-193. doi: 10.11648/j.js.20190706.17

Received: November 19, 2019; Accepted: November 29, 2019; Published: December 10, 2019

\begin{abstract}
Introduction: Incisional Hernia $(\mathrm{IH})$ is a frequent complication of abdominal surgery, with an incidence of 10$23 \%$, which can increase to $38 \%$ in specific risk groups. So far there is no ideal method to identify patients at high risk of developing $\mathrm{IH}$, this variety in the criteria of inclusion of patients in different studies makes is very complicate to compare the results. The aim of this study is comparing the two predictive scores of incisional hernias (Hernia project Vs Van Ramshorst) with higher diagnostic performance and determine which is better to predict IH. Methods: An analytical, observational study was conducted between June and December 2018, in patients of both sexes, who were 18 years or older, undergoing midline laparotomy, regardless of their background diagnosis either urgently or scheduled between 2007 to 2016 . The two different classifications, the Hernia Project score and Van Ramshorst score, were applied to each of the patients. With the identification of patients with hernia, two groups, healthy and ill, were performed and Bayesian statistics were made with it and to identify which scale best predicts the presence of this complication. Results: The records of 1085 patients undergoing midline laparotomy were reviewed. 296 patients were ruled out because their follow-up was not complete. Both tests were compared to determine the best diagnostic performance using the ROC curve and the area under the curve, finding that the Hernia Project Score has a larger area 0.724 compared to a 0.663 of Van Ramshorst. Discussion: In our patient cohort, the Hernia Project score has a greater predictive capacity, with an area under the best curve $(0.72$ vs. 0.66$)$ however both They have a poor sensitivity and this is the main measure of the predictive capacity of any diagnostic test, so with these results, we can affirm that the Project hernia scale has a greater diagnostic capacity than the Van Ramshorst scale but still its diagnostic capacity is limited. Conclusions: Both scores have low sensitivity. We can affirm that the Hernia Project score has a greater diagnostic capacity than the Van Ramshorst score, but both have a limited diagnostic capacity.
\end{abstract}

Keywords: Predictive Scores, Incisional Hernia, Hernia Project, Van Ramshorst Score

\section{Introduction.}

Incisional Hernia (IH) is a frequent complication of abdominal surgery, with an incidence of $10-23 \%$, which can increase to
$38 \%$ in specific risk groups [1]. IH can cause morbidity (eg, pain) and can have a negative effect on patients' quality of life and body image [2]. The substantial economic impact of the condition, estimated to be $>\$ 3$ billion annually in the US, is 
garnering more attention. Hernia prevention can come in many forms, including patient prehabilitation, optimal suture technique, and mesh reinforced closures. Patient Prehabilitation including control of diabetes, smoking cessation, weight loss, and good overall nutritional status, although in emergency surgery this is not possible [3]. The prevention of IH is important because of its high complication rate and whilst repair is possible recurrence rates are high, $32 \%$ with mesh repair.

In patients at elevated risk for $\mathrm{IH}$, prophylactic mesh implantation reduces the incidence of hernia formation, but with increased early postoperative pain and prolonged wound healing of surgical site infection [2, 4-9]. To maximize the benefit-risk ratio, despite technical modifications or prophylactic mesh implantation would ideally be performed in patients at elevated risk for hernia development. Previous studies explored the outcome of IH after specific procedures with elevated risk such as bariatric or abdominal vascular surgery. A population at risk was defined by patient-related risk factors that can be recognized preoperatively. Such stratification allows identification of patients at elevated risk for IH development, who potentially benefit the most from mesh implantation or despite technical modifications [4]. Deerenber et al. 2015 in the STITCH project included patients aged 18 years or older and scheduled to undergo abdominal surgery through a midline incision without identifying any extra risk factor [1]. Lozada et al (2016) in a clinical trial where they compare the usual closure versus reinforced tension line, they used the scale proposed by Van Ramshorst et al (2010) to identify high- risk patients as a criterion for inclusion in the experimental group, however the diagnostic performance was not $\operatorname{good}(10,11)$. Jairam et al $(2017)$ in the PRIMA trial including patients with abdominal aortic aneurysm and BMI $>$ 27 (2). Kohler et al (2018) used for stratification the following risk factors: overweight or obesity, diagnostic of neoplasic disease, male sex and history of laparotomy, patients with at least 2 of these risk factors were included [4].

So far there is no ideal method to identify patients at high risk of developing $\mathrm{IH}$, this variety in the criteria of inclusion of patients in different studies makes is very complicate to compare the results. Therefore, in the case of attempts to prevent incisional hernia formation, preoperative risk stratification and decision algorithms will be of potentially important value [3]. Recently, several investigators identified a multitude of risk factors and created risk models for predicting IH after surgery. [11-17]. The aim of this study is comparing the two predictive scores of incisional hernias (Hernia project Vs Van Ramshorst) with higher diagnostic performance and determine which is better to predict IH.

\section{Methods}

An analytical, observational study was conducted between June and December 2018, in patients of both sexes, who were 18 years or older, undergoing midline laparotomy, regardless of their background diagnosis either urgently or scheduled between 2007 to 2016 in the Hospital Regional de Alta Especialidad del Bajio, Mexico. This study was approved by the hospital's Ethics
Committee. Data collected in statistics report a total of 1089 patients susceptible to being subjected to the study.

\subsection{Instruments}

The two different classifications, the hernia project score and Van Ramshorst score, were applied to each of the patients. The Hernia score was published in 2015 , subsequently validated and corrected in 2017, is an assessment chart (table 1), which evaluate four criteria giving each one a value: 1 point for BMI > $25 \mathrm{~kg} / \mathrm{m}^{2}, 1$ point for COPD, 5 points for extended laparoscopy, 6 points for laparotomy and 3 points for previous abdominal operation. The sum of these factors gives rise to three stratified groups. Low risk 0 to 6.9 points, Medium risk 7 to 9 points and high risk was assigned $>9$ points.

The Van Ramshorst score has 10 variables; this score analyzes preoperative (age, Male gender, Chronic pulmonary disease, ascites, Jaundice, Anemia) Transoperative (Emergency surgery, type of surgery) and postoperative (coughing and wound infection) factors. With a maximum score of 10.6. (table 1). High-risk patients, for instance with scores of 6 or higher without counting postoperative factors such as coughing and wound infection, have a probability of developing abdominal wound dehiscence of more than $13.5 \%$. The risk model has shown high predictive value for the occurrence of incisional hernia in the validation analysis.

Incisional hernia: We used the definition of incisional hernia from the European Hernia Society (EHS) "any abdominal wall gap with or without bulge in the area of a postoperative scar perceptible or palpable by clinical examination or imaging. Or who have been operated for incisional hernia repair.

Table 1. Van Ramshorst and Hernia project scores.

\begin{tabular}{ll}
\hline Van Ramshorst Score & \\
\hline Variable & Risk score \\
\hline Age Category, years & \\
$40-49$ & 0.4 \\
$50-59$ & 0.9 \\
$60-69$ & 0.9 \\
$>70$ & 1.1 \\
Male Gender & 1.1 \\
COPD* & 1.1 \\
Ascites & 1.5 \\
Jaundice & 0.5 \\
Anemia & 0.7 \\
Emergency surgery & 0.6 \\
Type of surgery & \\
Gallbladder/bile duct & 0.7 \\
Esophagus & 1.5 \\
Gastroduodenum & 1.4 \\
Small bowel & 0.9 \\
Large bowel & 1.4 \\
Vascular & 1.3 \\
Coughing & 1.4 \\
Wound Infection & 1.9 \\
Hernia Project & \\
1 x (BMI $\left.\geq 25 \mathrm{Kg} / \mathrm{m}^{2}\right)+1 \mathrm{x}(\mathrm{COPD} *)$ & \\
+ (5x extended laparoscopy**) & \\
$+(6 x$ laparotomy) +3 (early abdominal Operation) \\
\hline
\end{tabular}

* Chronic Pulmonary disease **Operations involving an extended extraction site (ie laparoscopy colectomy, hand-assisted colectomy) 


\subsection{Process Description}

1. Initially, the statistical service was consulted to identify patients who met the inclusion criteria and a total population of 1089 patients is the target population.

2. The researchers reviewed the clinical file by calculating both scales for each patient and identified those with a diagnosis of incisional hernia.

3. With the identification of patients with hernia, two groups, healthy and ill, were performed and Bayesian statistics were made with it and to identify which scale best predicts the presence of this complication.

\subsection{Statistical Analysis}

A descriptive statistical analysis of the demographic data of the population was completed. It was carried out with the help of the commercial program SPSS 25 for Windows descriptive statistics to know the distribution of the variables. Two groups were formed according to the presence or not of incisional hernia, the continuous variables were reported as mean and standard deviation and the comparison of the results between both groups was carried out by means of a student $\mathrm{t}$ test for independent groups, complications and variables. Demographics were expressed as frequency and percentage; the comparison of the results was made through an $\chi^{2}$ test and Fisher's exact test for the different cases. In cases where it did not meet the normality assumptions, nonparametric statistics will be used with the Mann-Whitney U test. Sensitivity, specificity, negative and positive predictive value, positive and negative likelihood ratio were performed for each of the scales and Bayesian statistics were performed. The area under the curve was calculated and both scales were compared to determine which has better for predicted incisional hernia. A value of $p<0.05$ was considered significant.

\subsection{Ethical Aspects}

The research protocol was submitted for evaluation by the HRAEB Research Committee, which was approved by it, and a letter of confidentiality was made by the researchers.

The present study complies with the requirements of the Helsinki II 1964 code, as it is a study where a risk-free intervention will be carried out, since the method to investigate had no implications on the patient's health and did not delay in any way the attention to which He was subdued. Patient data was handled under strict confidentiality and privacy.

\section{Results}

The records of 1085 patients undergoing midline laparotomy were reviewed with the initial objective of identifying the presence of incisional hernia from 2007 to June 2018. 156 patients were ruled out because they died without completing the follow-up and 140 because they were discharged, and their follow-up was not complete. A total of 789 patients with an average age of $49.67 \pm 15.91$ years were analyzed, of which $263(33.4 \%)$ were men and $526(66.6 \%)$ were women. Of the total laparotomies $173(21.9 \%)$ were with medical emergency criteria and $582(73.9 \%)$ had a background cancer diagnosis, this explained by the characteristics of the model of care of our hospital. Of these patients, 161 were found to have incisional hernia (20.4\%).

The next objective of the work was to compare two diagnostic scores as predictors of the presence of incisional hernia. The first to be analyzed was the Van Ramshorst score (2010) The variables that make up the score were analyzed separately and then their final score globally, two groups were formed, those with and without incisional hernia; finding statistically significant difference between both groups in the age of laparotomy (0.009), the presence of anemia $(0.045)$, if it was emergency surgery $(0.0001)$, the post-surgical presence of cough (0.001) and surgical site infection (0.0001).

The overall score of patients with incisional hernia had a median of 3 with a $25-75 \%$ percentile of $0.9-4.25$ compared to non-herniated patients with a median of $2.1(0.9-3)$ with a statistically significant difference between the two groups (0.005). Table 2.

Table 2. Van Ramshorst Score.

\begin{tabular}{llll}
\hline Variable & Hernia n=161 & $\begin{array}{l}\text { Without Hernia } \\
\mathbf{n = 6 2 7}\end{array}$ & $\mathbf{p}$ \\
\hline Age & $52.37 \pm 14.41$ & $48.94 \pm 16.22$ & $0.009^{*}$ \\
Gender & & & \\
Female & $98(60.9 \%)$ & $428(68.2 \%)$ & $0.092^{* *}$ \\
Male & $63(39.1 \%)$ & $200(31.8 \%)$ & \\
Type of surgery & & & \\
Gallbladder/bile duct & $15(9.3 \%)$ & $64(10.2 \%)$ & \\
Esophagus & $8(4.9 \%)$ & $38(6 \%)$ & \\
Gastroduodenum & $33(20.4 \%)$ & $119(18.9 \%)$ & $0.654^{* * * *}$ \\
Small bowel & $51(31.6 \%)$ & $220(35 \%)$ & \\
Large bowel & $48(29.8 \%)$ & $171(27.3 \%)$ & \\
Vascular & $6(3.7 \%)$ & $15(2.4 \%)$ & $0.544^{* *}$ \\
COPD & $4(2.5 \%)$ & $11(1.8 \%)$ & $0.400^{* *}$ \\
Ascites & $18(11.2 \%)$ & $37(5.9 \%)$ & $0.464^{* *}$ \\
Jaundice & $12(7.5 \%)$ & $37(5.9 \%)$ & $0.045^{* *}$ \\
Anemia & $20(12.4 \%)$ & $47(7.5 \%)$ & $0.0001^{* *}$ \\
Emergency surgery & $55(34.2 \%)$ & $118(18.8 \%)$ & $0.0001^{* *}$ \\
Cough & $25(15.5 \%)$ & $37(5.9 \%)$ & $0.0001^{* *}$ \\
Surgical Site & $55(34.2 \%)$ & $56(8.9 \%)$ & $0.0005^{* * *}$ \\
Infection & $3(0.9-4.25)$ & $2.1(0.9-3)$ & \\
Overall Score &
\end{tabular}

*Quantitative variable is reported as mean and standard deviation and the comparison between groups was performed with a student $t$ test for independent groups.

**Qualitative variables are reported as frequency and percentage and the comparison between groups was performed with an exact Fisher test.

***Quantitative variable is reported as median and $25-75 \%$ percentile and the comparison between groups was performed with a Whitney U-Mann test. ****Qualitative variables are reported as frequency and percentage and the comparison between groups was performed with $\chi^{2}$ test.

The final rating of the score determines the possibility of incisional hernia being high risk those patients with score greater than 6 . For this score a sensitivity of $27 \%$ and specificity of $92 \%$ was found when compared with the gold standard Table 3. 
Table 3. Comparison of both scores in their diagnostic efficiency in high risk patients.

\begin{tabular}{llllll}
\hline Score & Sensitivity & Especificity & PPV* & NPV** & LR+*** \\
\hline Van Ramshorst & $27.3 \%$ & $92.7 \%$ & $50 \%$ & $82.5 \%$ & 3.77 \\
Hernia score & $74.53 \%$ & $59.3 \%$ & $32 \%$ & $90 \%$ & 0.78 \\
\hline
\end{tabular}

*PPV: positive predictive value **Negative predictive value $* * * \mathrm{LR}+$ positive likelihood ratio.

$* * * *$ Negative likelihood ratio.

The Hernia Project score was evaluated, and its measurements were compared separately and in overall percentage, a statistically significant difference was found in cases of having received a midline laparotomy (0.0001), extended laparoscopy (0.0001), having had previous surgery (0.0001) and body mass index greater than $25(0.008)$, having no effect having been diagnosed with COPD Table 4.

Table 4. Hernia Project score.

\begin{tabular}{llll}
\hline Variable & Hernia $\mathbf{n = 1 6 1}$ & Without hernia $\mathbf{n = 6 2 7}$ & P \\
\hline Midline laparotomy & $131(81.4 \%)$ & $396(63.1 \%)$ & $0.001^{*}$ \\
Extended laparoscopy & $28(17.4 \%)$ & $218(34.7 \%)$ & $0.0001^{*}$ \\
Prior surgery & $126(78.3 \%)$ & $293(46.7 \%)$ & $0.0001^{*}$ \\
COPD & $4(2.5 \%)$ & $11(1.8 \%)$ & $0.544^{*}$ \\
BMI $>25 \mathrm{~kg} / \mathrm{m}^{2}$ & $28.9 \pm 17.6$ & $26.1 \pm 10.4$ & $0.008^{* *}$ \\
Overall score & $9(8-10)$ & $7(6-9)$ & $0.0001^{* * *}$ \\
\hline
\end{tabular}

*Qualitative variables are reported as frequency and percentage and the comparison between groups was performed with an exact Fisher test.

**Quantitative variable is reported as mean and standard deviation and the comparison between groups was performed with a student $\mathrm{t}$ test for independent groups.

*** Quantitative variable is reported as median and 25-75\% percentile and the comparison between groups was performed with a Whitney U-Mann test.

The final rating of the score determines the possibility of incisional hernia as low of 0-6.99, intermediate of 7-9 and more than 9 high risk, the diagnostic performance of the score was calculated against the definitive diagnosis Table 3.
Both tests were compared to determine the best diagnostic performance using the ROC curve and the area under the curve, finding that the Hernia Project Score has a larger area 0.724 compared to a 0.663 of Van Ramshorst Figure 1.

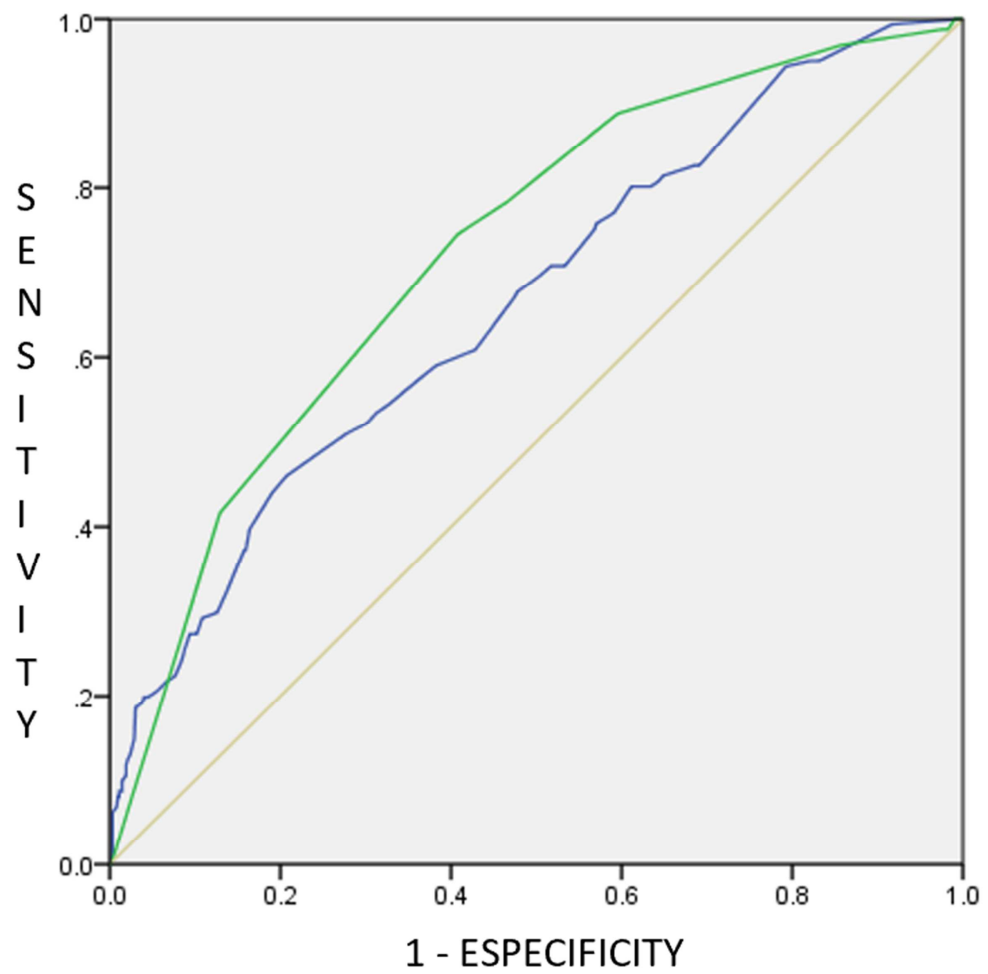

\section{- VAN RAMSHORT - HERNIA PROJECT REFERENCE LINE}

Figure 1. ROC curve comparison.

When analyzing the characteristics of patients with incisional hernia, we identify variables associated with the presence of this complication, which are not evaluated in the two scores but reported in the literature, a statistically significant difference was found due to having had an early relaparotomy, the presence of stoma and having a larger 
incision at $18 \mathrm{~cm}$ they had an association with it; not so having an oncological pathology in its diagnosis Table 5.

Table 5. Factors associated with incisional hernia.

\begin{tabular}{lllll}
\hline Variable & Hernia $\mathbf{n = 1 6 1}$ & Without hernia n=627 & P & OR (CI-95\%)* \\
\hline Early relaparotomy & $87(54 \%)$ & $129(20.5 \%)$ & 0.0001 & $4.5(3.14-6.54)$ \\
Oncological diagnosis & $110(68.3 \%)$ & $472(75.2 \%)$ & 0.079 & NS \\
Stoma & $56(34.8 \%)$ & $124(19.7 \%)$ & 0.0001 & $2.46(1.48-3.16)$ \\
Wound greater than $18 \mathrm{~cm}$ & $108(67.1 \%)$ & $345(55 \%)$ & 0.018 & $1.66(1.15-2.39)$ \\
\hline
\end{tabular}

Qualitative variables are reported as frequency and percentage and the comparison between groups was performed with an exact Fisher test.

$* \mathrm{OR}=$ odds Ratio $\mathrm{CI}=$ Confidence Intervals $-95 \%$.

\section{Discussion}

The purpose of our study was to compare the ability of two clinical scoring systems, the Van Ramshorst score and Hernia project to diagnose or rule out incisional hernia.

$\mathrm{IH}$ is a common pathology and is the main complication after midline laparotomy and its presence is associated with high morbidity, decreased quality of life and high costs in hospital care. The incidence of incisional hernia is around 15$20 \%$ and can reach up to $50 \%$ in emergency or high-risk patients $[1,2]$; In our cohort we found an incidence of $20.4 \%$, within the reported international average.

The appearance of an incisional hernia is multifactorial, about 30 risk factors have been described, which is why in recent years the literature is focused on the prophylaxis of the incisional hernia to reduce this incidence. Prophylaxis strategies range from the modification of the surgical technique, as reported in the study by Lozada et al. In 2016 [10], in which an abdominal wall reinforcement was performed with the RTL technique, reducing the presence of this complication from 20 to $4.5 \%$. Most current studies are focused on the use of mesh prophylactically and report an overall incidence with its use of $9 \%$ [2, 4-9].

Although all these strategies have proven useful in reducing the incidence of incisional hernia, there is still no clear consensus as to who, and in what position they should be placed a mesh, or in whom to modify the surgical technique, the most of the literature only recommends it for patients with a high risk of incisional hernia. However, the problem is to define who these patients are at high risk, so the objective of this study was to determine if there is any score that allows determining the possibility of incisional hernia and classify patients as high or low possibility of presenting this complication.

Webster and collaborators in 2003 [12], devised a score to determine high-risk patients, however they took 12 variables in their scale, the problem is that 6 of them occurred in the postoperative period, and this decreased their predictive capacity.

In 2010 Van Ramshorst and collaborators [11] reported a scale subsequently validated by Gomez and collaborators [14] in which they took into account 9 variables, finding that patients with a score greater than 6 were high risk, with a possibility of herniation of the $40 \%$, however, when doing its postoperative evaluation, only a $16 \%$ occurrence was found, so its predictive capacity is poor. However, it should be noted that in the validation study only patients with acute evisceration were reviewed.

In our study we analyzed the predictive capacity of the score in a patient with 2 years of follow-up, we found that its sensitivity and specificity is 27.3 and $92.7 \%$ respectively, with an area under the curve of $0.66 \%$ which makes according to these results a limited utility in your prediction.

We also evaluated the Hernia Project score in 2015 [15] and later validated in 2017 [16] in which they take into account 5 variables, finding that patients with a greater than 9 were considered high risk, with a possibility of diagnosis of hernia incisional of $28.8 \%$ clinical and radiological reported up to $57.5 \%$.

The predictive score of this scale was analyzed in the same way with 2 years of follow-up finding a sensitivity and specificity of 74.53 and 59.3 respectively with an area under the curve of 0.72 .

The main objective of the study was to compare the predictive capacity of both scores, we found that in our patient cohort, the Hernia Project scale has a greater predictive capacity, with an area under the best curve $(0.72$ vs. 0.66 ) however both they have a poor sensitivity and this is the main measure of the predictive capacity of any diagnostic test, so with these results, we can affirm that the Project hernia score has a greater diagnostic capacity than the Van Ramshorst scale but still its diagnostic capacity is limited

As strength of our study we can mention 2 things, one, the number of patients that were taken into account for the evaluation (789), two the 2-year follow-up according to what the EHS guidelines recommend in the which, it recommends that any study that evaluated the presence of instructional hernia, should take at least 2 years follow-up. The study is significant, so they could be studied in future work.

According to the results of Jairam and cols 2017, where they found a prevalence a priori of $30 \%$ of incisional hernias when primary closure is performed only, the prevalence calculated a posteriori using Bayesian statistics, finding that both scores are bad to predict Figure 2 .

As a weakness we find that we do not have the exact time in which this complication was found, as well as external validity, since our hospital is a third level hospital and in our service portfolio we do not have emergency services, but Chronic patients with a background cancer diagnosis predominate, so our findings may not be transpolated to that of the largest health institutions in the country.

We found that the scores only coincide in a variable that is COPD, both have been validated with many patients so making a new scale complementing the 2 , we believe it is a 
very good opportunity area for further studies. This, in addition to the fact that during the study the oncological diagnosis of the patients, early relaparotomy, presence of stoma and a wound larger than $18 \mathrm{~cm}$ were independently assessed, variables of which, all except the oncological diagnosis have statistically significant differences, so they could be studied in future work.

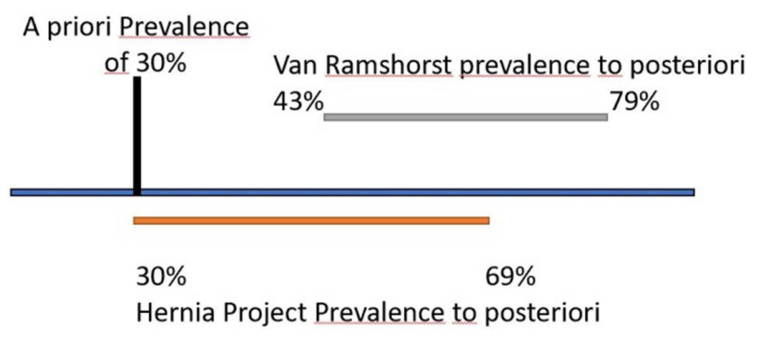

Figure 2. Bayesian statistics for calculate to prevalence posteriori.

\section{Conclusions}

In our cohort there is an incidence of $20.4 \%$ of incisional hernias. When comparing the predictive capacity of both scales, we found that in our patient cohort, the Hernia Project score has a greater predictive capacity, with an area under the better curve (0.72 vs. 0.66), however both have poor sensitivity and that this it is the main measure of the predictive capacity of any diagnostic test, so with these results, we can state that the Hernia Project escore has a greater diagnostic capacity than the Van Ramshorst scale but still its diagnostic capacity is limited.

\section{References}

[1] Deerenberg EB, Harlaar JJ, Steyerberg EW, et al. Small bites versus large bites for closure of abdominal midline incisions (STITCH): a double-blind, multicenter, randomized controlled trial. Lancet 2015; 386: 1254-1260.

[2] Jairam AP, Timmermans L, Eker HH, et al. PRIMA Trialist group. Prevention of incisional hernia with prophylactic onlay and sublay mesh reinforcement versus primary suture only in midline laparotomies (PRIMA): 2-years follow-up of a multicenter, double blind, randomized controlled trial. Lancet. 2017; 390 (10094): 567-576. doi: 10.1016/S0140-6736(17) 31332-6.

[3] H. W. Harris et al., Contemporary concepts in hernia prevention: selected proceedings from the 2017 international simposium of prevention of Incisional Hernia. Surgery 2018. http://doi.org/10.1016/j.surg.2018.02.020.

[4] Kohler A, Lavanchy JL, Lenoir U, Kurmann A, Candinas D, Beldi G. Effectiveness of prophylactic intraperitoneal mesh implantation for prevention of incisional hernia in patients undergoing open abdominal surgery A Randomized Clinical Trial. JAMA surgery 2019. 154 (2): 109-115.
[5] Caro-Tarrago A, Olona C, Millan M, Olona M, Espina B, Jorba R. Long-term results of a prospectiv randomized trial of midline laparotomy closure with onlay mesh. Hernia (2019) 23: $335-340$.

[6] Hernandez-Granados P, Lopez-Cano M, Morales-Conde S, Muysoms F, Garcia-Alamino J, Pereira-Rodriguez JA Profilaxis de la hernia incisional y utilización de mallas. Revision Narrativa. Cir Esp 2018; 96 (2): 76-87.

[7] Glauser PM, Brosi P, Speich B, Käser SA, Heigl A, et al. Prophylactic intraperitoneal onlay mesh following midline laparotomy-Long-term Results of a Randomized Controlled Trial. World J Surg 2019; 43 (7): 1669-1675. Doi: 10.1007/s00268-019-04964-6.

[8] San Miguel C, Melero D, Jimenez E, Lopez P et al. Long-term outcomes after prophylactic use of onlay mesh in midline laparotomy. Hernia (2018); 22: 1113-1122. doi: http://doi.org/10.1007/s10029-018-1833-x.

[9] Payne R, Aldwinckle J, Ward S. Meta-analysis of randomized trials comparing the use of prophylactic mesh to standard midline closure in the reduction of incisional herniae. Hernia 2017. 21: 843-853.

[10] Lozada-Hernandez EE, Mayagoitia-Gonzalez JC, SmolinskiKurek R, Alvarez-Canales JJ, Montiel-Hinojosa L et al. Comparación de dos técnicas de sutura para cierre aponeurotico en laparotomía media en pacientes con alto riesgo de evisceración posquirúrgica. Rev Hispanoam Hernia. 2016; 4 (4): 137-143.

[11] Van Ramshorst GH, Nieuwenhuizen J, Jop WC, Arends P, Boom $\mathrm{J}$ et al. abdominal wound dehiscence in adults: development and validation of a risk model. World $\mathrm{J}$ surg. 2010; 34: 20-7.

[12] Webster C, Neumayer L, Smout R, Horn S, Daley J et al. Prognostic models of abdominal wound dehiscence after laparotomy. Journal of surgical Research (2003); 109: 130137. doi: $10.1006 /$ jsre.2003.660.

[13] Veljkovik R, Protic M, Gluhovic A, Potic Z, Milosevic A et al. Prospective clinical trial of factors predicting the early development of incisional hernia after midline laparotomy. J Am Coll Surg 2010; 210: 201-219.

[14] Gómez-Diaz CJ, Rebasa-Cladera P, Navarro-Soto S, HidalgoRosas JM, Luna Aufory A et al. Validación de un modelo de riesgo de evisceración. Cir Esp. 2014; 92 (2): 114-119.

[15] Goodenough CJ, Ko CT, Kao LS, Nguyen MT, Holihan Jl et al. Development and validation of a risk stratification score for ventral hernia after abdominal surgery: Hernia expectation rates in intra-abdominal surgery (The HERNIA Project). J Am Coll Surg 2015; 220: 405-413.

[16] Cherla DP, Moses ML, Muek KM, Hannon C, Ko CT et al. External validation of the HERNIA score: an observational study. J Am Coll Surg 2017; 225: 428-434.

[17] Fischer JP, Basta MN, Mirzabeigi MN, Bauder AR, Fox JP, Drebin JA, et al. A risk model and cost analysis of incisional hernia after elective abdominal surgery based upon 12373 cases: the case for targeted prophylactic intervention. Ann Surg 2016; 263: 1010-1017. 